\title{
The Mechanical Performance Evaluation of Vertical Stability Coil under Electro- magnetic-structure Coupling Analyses
}

\author{
Peng Han ${ }^{1}$, Xianewei Wang ${ }^{2}$ \\ ${ }^{1}$ North Information Control Research Academy Group Co.,Ltd, Nanjing, 211100, China. E-mail: \\ 859313246@qq.com \\ 2Jiangsu University of Technology, Changzhou 213001, China., E-mail: wangxw@jsut.edu.cn
}

The vertical stability coil is a set of active feedback control coil that is used to deal with the vertical instability of plasma. To improve the response performance, the coil is mounted in the vacuum vessel, which denotes the coil-body will suffer from large electromagnetic force from the plasma current and poloidal field coils. Besides the current flowing in the feeder is nearly perpendicular with magnetic field originated from toroidal coil. It implies large electromagnetic force will be generated on the feeder. In order to withstand the impact from the electromagnetic force, a series of reinforce components are designed and installed on the coil. It is necessary to verify whether or not the coil conductor and auxiliary components could successfully bear the shock of large electromagnetic force. A three-dimensional magnetic field model is built to accurately calculate the magnetic field and electromagnetic force. Corresponding to the magnetic field calculation model, a more detailed me-chanical analysis model is created to launch the electromagnetic-structural coupling analysis. Based on the stress analysis results, the local structure of the coil is optimized to decrease the peak stress. The updated model is reanalyzed and stress linearization is exerted to extract the different kinds of stress on the coil components. Finally, the stress is evaluated based on ASME analytical design. The evaluation result is helpful to guide the further design optimization.

Keywords: Vertical stability, Magnetic field, electromagnetic force, Stress evaluation

\section{Introduction}

The plasma in the fusion device is inherently unstable. Thus, the In-Vessel coils are proposed to solve the prob-lem. The In-Vessel coils mainly contains the Edge Localized Mode coil (ELM) and Vertical Stability (VS) coil. And the main function of VS coil is to improve the vertical stability of plasma. In order to avoid the magnetic shielding and achieve better response performance, the VS coil is mounted in the vacuum vessel. The VS coil contains two sets of coil, namely the upper and lower VS coil, which are connected in series and have the saddled shape as shown in Fig. 1. In view of the severe operation environment, the Stainless Steel Mineral Insulation Conductor is used for the coil $[1,2,3]$. The coil is composed of three different layers, they are the inner hollow copper conductor, the middle compacted Magnesium Oxide $(\mathrm{MgO})$ ring and the outer stainless steel. The func-tions of the copper conductor are carrying the large current and providing the channel for cooling water. The $\mathrm{MgO}$ is not only a good electrical insulation material but also acts excellently at enduring the large dose of neu-tron radiation $[4,5,6]$. The stainless steel works as a protection layer to prevent the possible performance degra-dation of $\mathrm{MgO}$. Besides the conductor-body there are also some auxiliary components such as the spine, clamp and rail, which are used to fix and support the conductor. The coil is finally welded to the vacuum vessel wall through the toroidal arranged supporting rail.

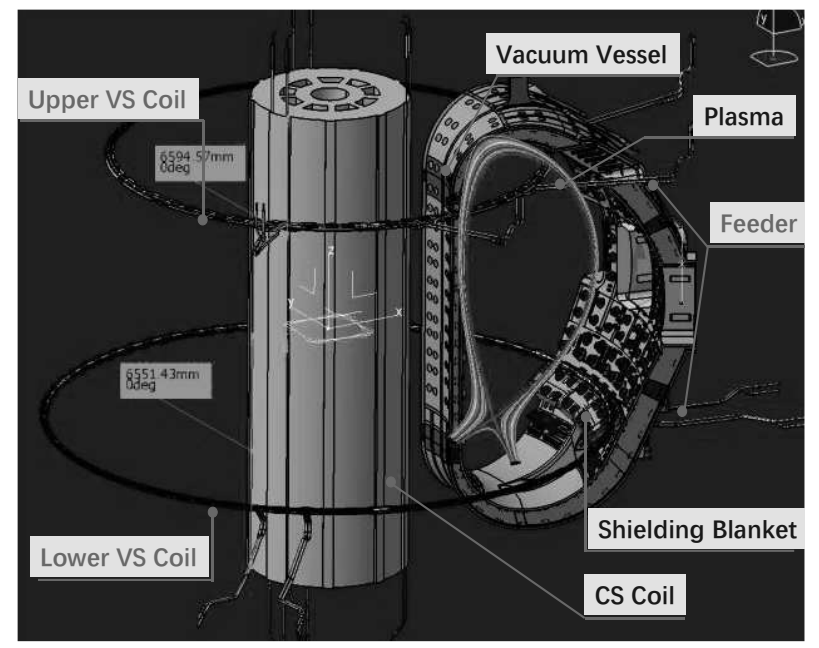

Fig. 1 The geometrical structure of VS coil

Once the coil is charged large electromagnetic force will be gener-ated on the coil body and leading out feeders. And the electromagnetic force may result in the local structural damage. Thus, it is necessary to carry out the mechanical analysis to verify whether or 
not the current structural design can withstand the impact of electromagnetic force. Note the fact that the thermal expansion coefficient of vacuum vessel is smaller than the VS coil, which means the circumferential compressive force will be generated. The compressive force is beneficial to inhibit the expansion of fatigue crack caused by the electromagnetic force. Thus, electromagnetic fatigue is not studied here. While the peak electromagnetic force as the worst load state which will significantly affect VS coil mechanical performance is analysed in detail in the paper.

\section{The electromagnetic analysis}

The key of electromagnetic analysis is to calculate the magnetic field. The magnetic field contains two aspects. One is from the VS coil self, the other is from the background field coils and plasma. The background field coils contain the Central Solenoid (CS) coil, Poloidal Field (PF) coil, Toroidal Field (TF) coil, Correction Field (CF) coil and Edge Localized Mode (ELM) coil. Since the currents of CF coil and ELM coil are far smaller than other coils. They are not considered in the magnetic field calculation. For the plasma, it is equivalent to current block with rectangular cross section [7,8]. The magnetic field of background field coil can be calculated based on different equivalent models such as current fillet equivalent model, discrete $\mathrm{N}$ turns current fillet equivalent model $(\mathrm{N}$ is the turns of the specific coil) and current block model [9]. Where we use the commercial software ANSYS Maxwell to calculate the magnetic field. And the Magnetic Vector Potential (MVP) calculation method is selected due to its succinct simulation model. The plasma current and background field coil are built as current source in the finite element model and there is no need to build the air field [10]. The detailed analysis model is shown in Fig. 2. The magnitude of magnetic field is related with the plasma operation scenarios. The magnetic analysis indicates the magnetic field will reach the peak value at the moment of End of Burn (EOB). Thus, where we choose the current of EOB moment to carry out the electromagnetic analysis. And the calculation result of magnetic field on the VS coil is shown in Fig. 3. For the convenience of describing the magnetic field. The different turns of VS coil are labelled as turn01, turn02, turn03R, turn03L and turn04 respectively.

The magnetic field calculation results indicate the magnetic field magnitude of the four turns are similar to each other. Because the conductors are arranged closely to each other. And the magnetic field mainly vary along the radial direction. The variation trend of magnetic field is that it first increases to a certain value, then begin to fluctuate around the value and finally quickly decrease to a small value. The increasing region represents the lead-in feeder. Since the conductor is gradually approaching the background field coil, the magnetic field on the feeder increases quickly. The fluctuation region is corresponding to the toroidal coil body. The magnetic field in the toroidal direction has tiny change because of the small distance variation between the VS coil and background field coil. And the distance variation is caused by the $\mathrm{S}$ bending and $\mathrm{W}$ bending arcs with changing curvature radii. The design of bending arcs is to prevent the interference between VS coil and other adjacent components. The magnetic field decreasing region represents the leadout feeder. Since the feeder is gradually off the background coil, the magnetic field on the feeder decreases simultaneously. In addition, the magnetic field of the lead-in and lead-out feeders is nearly symmetric, which is because the feeders are closely bound together. The minimum magnetic field occurs at the end of the feeder and the value is about $0.5 \mathrm{~T}$. The peak magnetic field on the VS coil is about $6.18 \mathrm{~T}$.

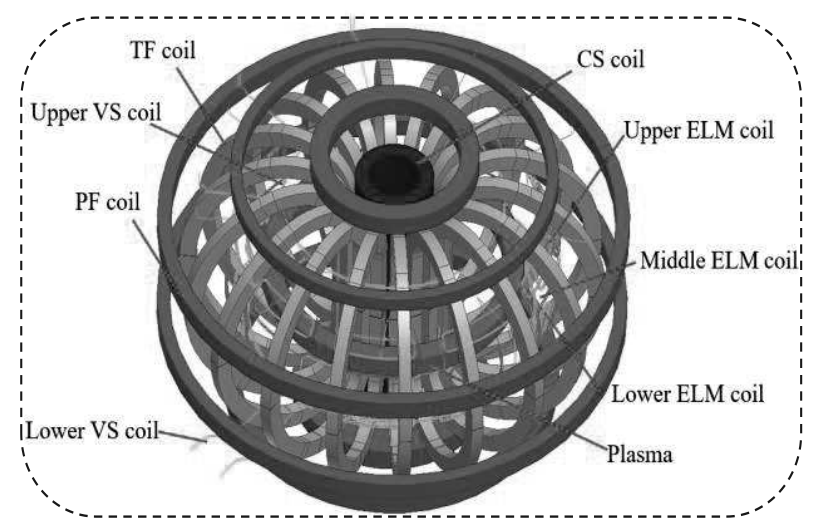

Fig. 2 The equivalent background coil and plasma current model

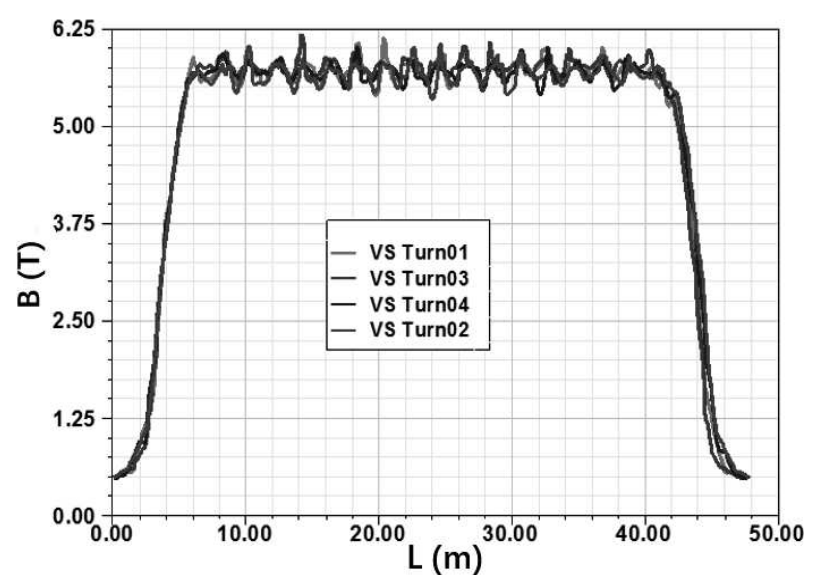

Fig. 3 The magnetic field on the VS coil

The electromagnetic force is calculated based on Ampere's Force Law. The peak current flowing through per turn of VS conductor is $60 \mathrm{kA}$, which is selected to perform the conservative analysis. The 
current direction is assumed in the anti-clockwise direction. Considering the approximately asymmetry of the VS coil in the circumferential direction, a $40^{\circ}$ model (shown in Fig. 4) is built to calculate the electromagnetic force. The detailed electromagnetic force density is shown in Fig. 5-9. The results indicate the electromagnetic force on the coil-body is smaller than that on the feeder. The electromagnetic force at the break-out region changes sharply due to the variation of background magnetic field. And the region near to the coil-body (small straight segments) has a large angle with background magnetic field, which will lead to the large electromagnetic force. While the region approaching the straight feeder (small toroidal arc) is nearly parallel with the toroidal magnetic field, which denotes the generated electromagnetic force will be very small. For the straight feeders, since they are away from the device centre, the electromagnetic force decreases quickly, which is constant with the variation of magnetic field. For the turn01 and turn02, they have the similar electromagnetic force distribution owing to the same structure. And the sum electromagnetic force didn't have large fluctuation due to the constant radius in the toroidal direction. For the turn03, the steep variation of electromagnetic force is caused by the break-out structural design and current feeders. For the turn04, the variation of sum electromagnetic force is related with the $\mathrm{S}$-bending structure.

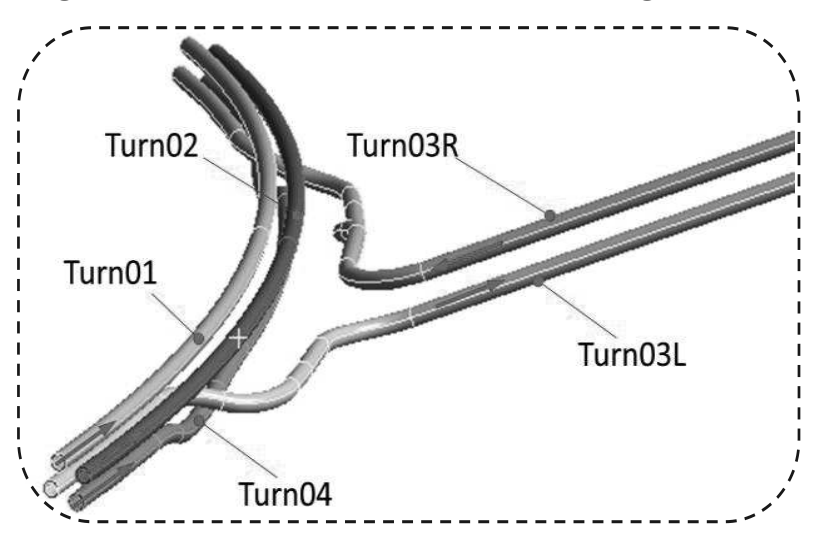

Fig. 4 The different turns of VS coil $\left(40^{\circ}\right.$ model)

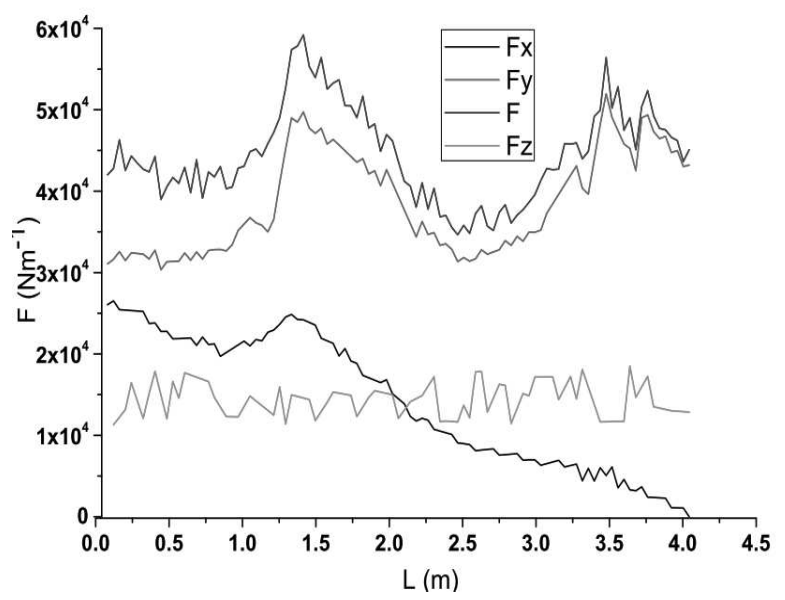

Fig. 5 The electromagnetic force density of turn 01

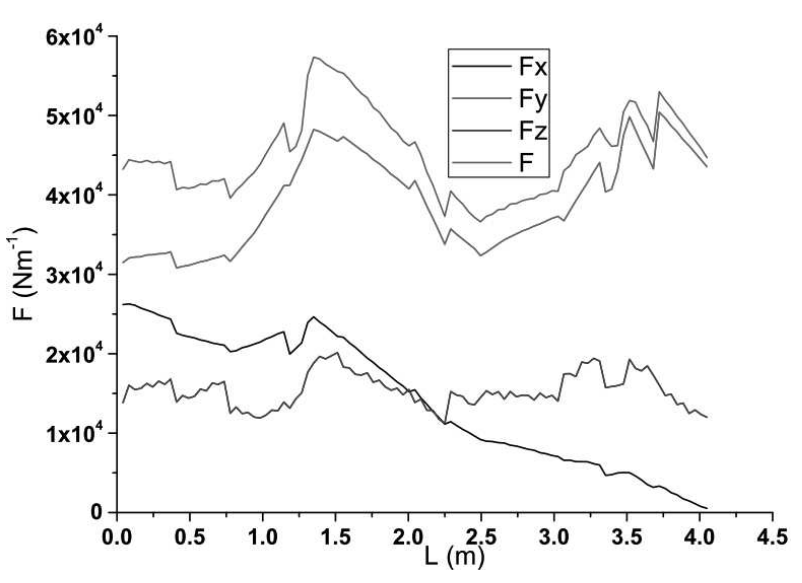

Fig. 6 The electromagnetic force density of turn 02

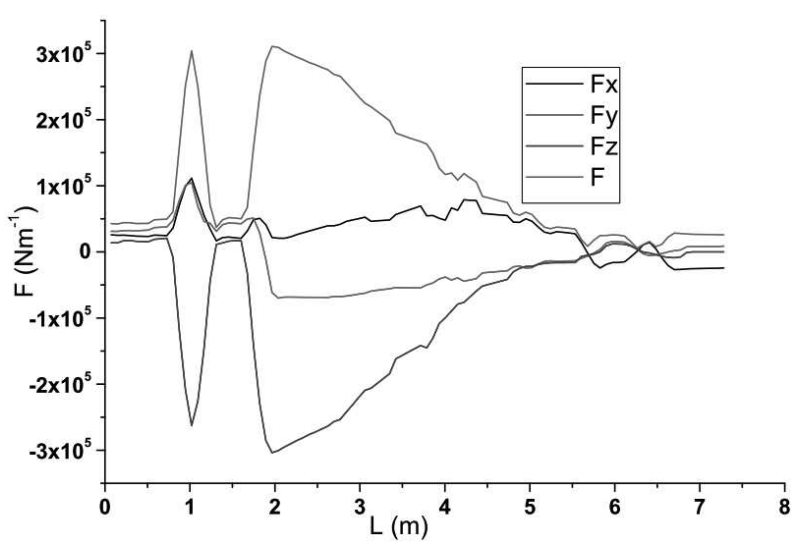

Fig. 7 The electromagnetic force density of turn $03 \mathrm{~L}$

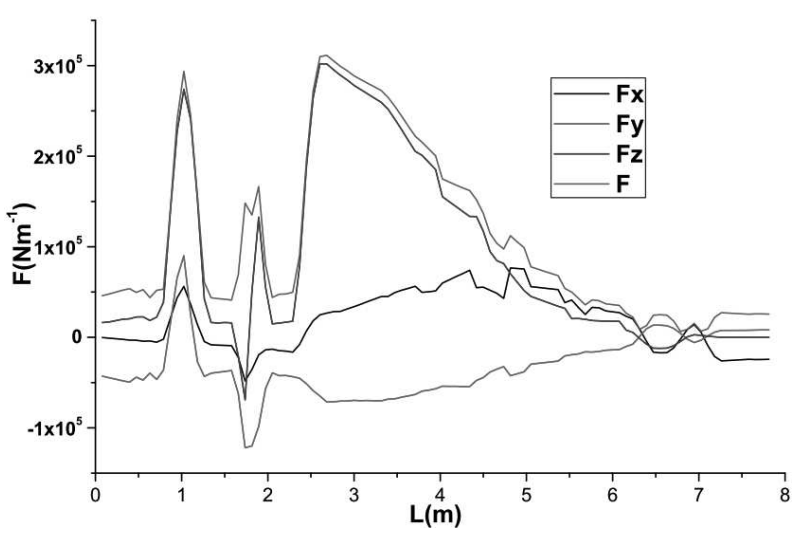

Fig. 8 The electromagnetic force density of turn $03 \mathrm{R}$

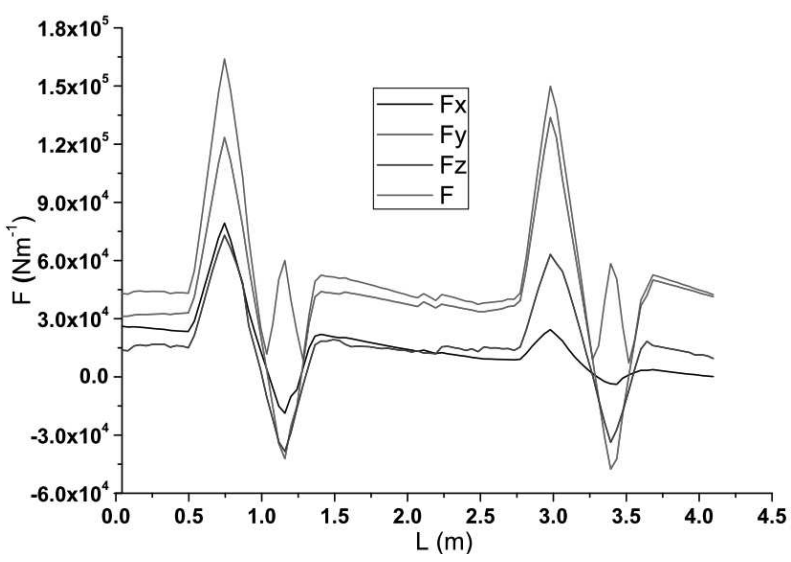

Fig. 9 The electromagnetic force density of turn 04 


\section{The electromagnetic-structural coupling analysis and stress evaluation}

3.1 The electromagnetic-structural coupling analysis

Corresponding to the electromagnetic analysis model, a $40^{\circ}$ mechanical analysis model is built. Besides the copper tube the remaining coil components such as $\mathrm{MgO}$, jacket, spine, clamp, gasket and rail are added in the model. The detailed model is shown in Fig. 10. The model is meshed with different meshing technology such as sweeping, mapping and multi-zone. The high-order element is used to improve the calculation accuracy [11]. Hexahedron element is selected to decrease the total elements. The total nodes of the model are about 9830,000. The boundary condition is applied as follow: The bonding contact is set between the conductor jacket and the supporting components. The bottom surface of rail is fixed. For the bracket components, their normal displacement and rotation are restrained. Moreover, the frictionless restraint is applied at the high and low boundaries of the finite element model. The electromagnetic force is input from the above electromagnetic analysis file. The material properties used in the structural analysis are listed in Table 1.

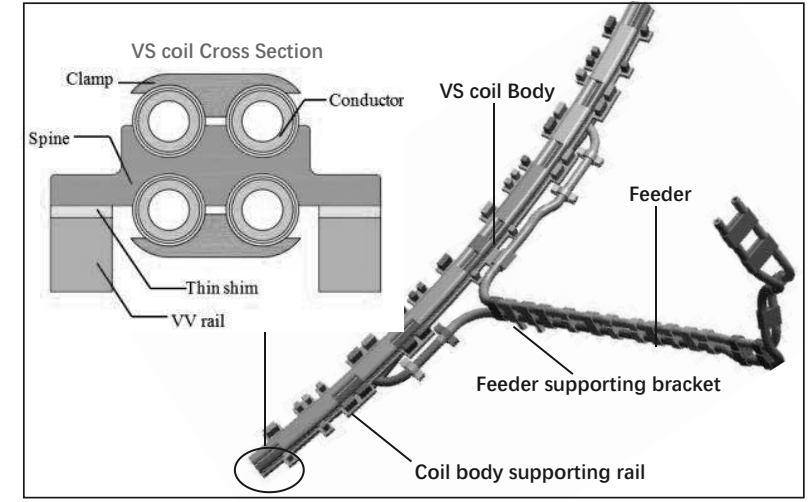

Fig. 10 The $40^{\circ}$ analysis model

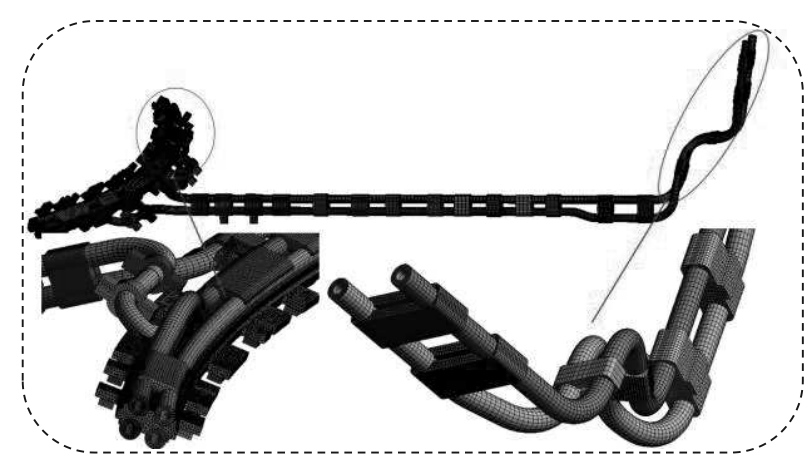

Fig. 11 The $40^{\circ}$ finite element model

Tab. 1 VS coil material properties under different temperatures

\begin{tabular}{|c|c|c|c|c|c|c|}
\hline Parameters & Stainless s & eel (316LN) & Copper & C10200) & $\mathrm{MgO}$ & Unit \\
\hline Temperature & 100 & 200 & 100 & 200 & - & ${ }^{\circ} \mathrm{C}$ \\
\hline Density & 8000 & 8000 & 8903 & 8854 & 2200 & $\mathrm{Kg} \cdot \mathrm{m}^{-3}$ \\
\hline Elastic modulus & $1.92 \mathrm{e} 11$ & $1.83 \mathrm{e} 11$ & $1.14 \mathrm{e} 11$ & $1.10 \mathrm{e} 11$ & $9.6 \mathrm{e} 8$ & $\mathrm{~Pa}$ \\
\hline Poisson's ratio & 0.298 & 0.306 & 0.33 & 0.33 & 0.3 & - \\
\hline $\begin{array}{l}\text { Coefficient of thermal } \\
\text { expansion }\end{array}$ & 16.8 & 17.2 & 17.2 & 17.59 & 12.8 & $10^{-6} \mathrm{~K}^{-1}$ \\
\hline $\begin{array}{l}\text { Coefficient of heat con- } \\
\text { duction }\end{array}$ & 15 & 16.6 & 395 & 388 & 2.36 & $\mathrm{~W} \cdot(\mathrm{m} \cdot \mathrm{K})^{-1}$ \\
\hline Specific heat & 489 & 523 & 394 & 401 & 940 & $\mathrm{~J} \cdot(\mathrm{Kg} \cdot \mathrm{K})^{-1}$ \\
\hline
\end{tabular}

The equivalent shear stress induced by electromagnetic force is shown in Fig. 12. The maximum stress is about $197 \mathrm{MPa}$, which occurs at the break-out region and is on the joint sleeve. The stress distribution on the copper conductor is shown in Fig. 13. The maximum stress is about $59 \mathrm{MPa}$, which occurs at the feeder straight segment. In order to mitigate the local large stress on the feeder, the supporting brackets are optimized. Firstly, the width of bracket at the breakout region is increased from $90 \mathrm{~mm}$ to $120 \mathrm{~mm}$. Then two extra brackets are added at the feeder straight segment as shown in Fig. 14. The stress analysis results based on the updated model are shown in Fig. 15-16. It indicates the peak stress on the jacket decreases about $37 \mathrm{MPa}$. The stress on the copper conductor decreases about $10 \mathrm{MPa}$.

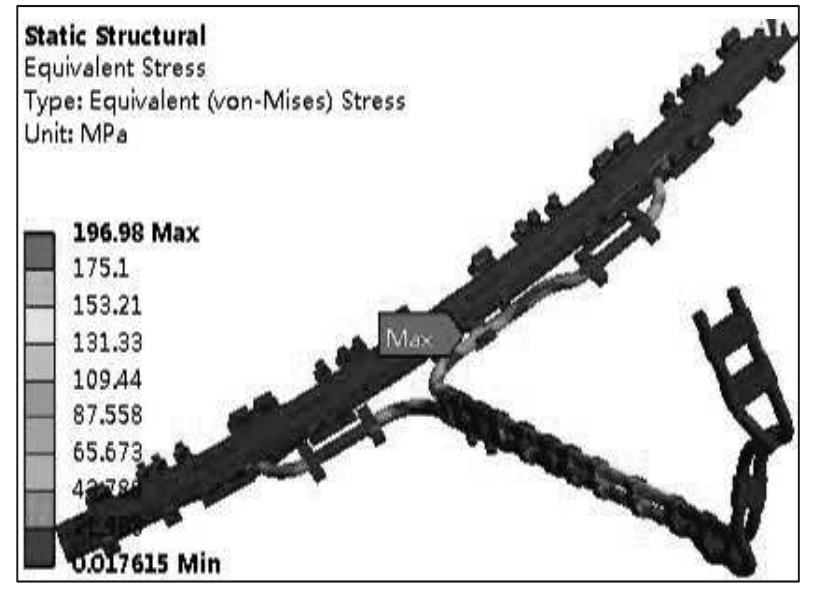

Fig. 12 The equivalent stress on the VS coil 


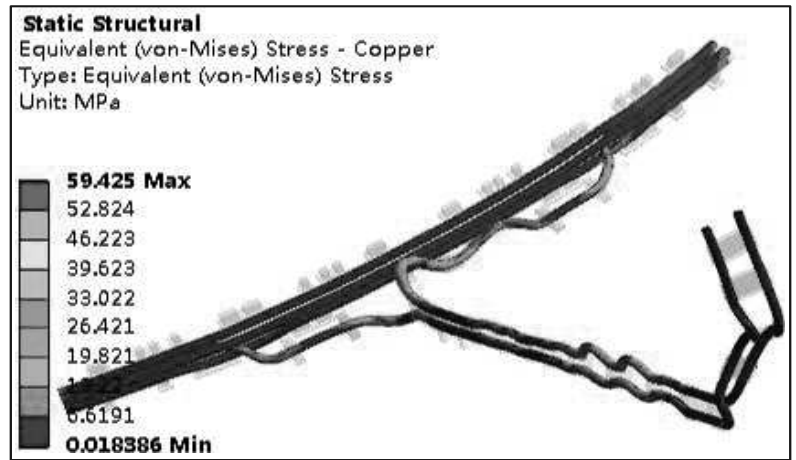

Fig. 13 The equivalent stress on the copper conductor

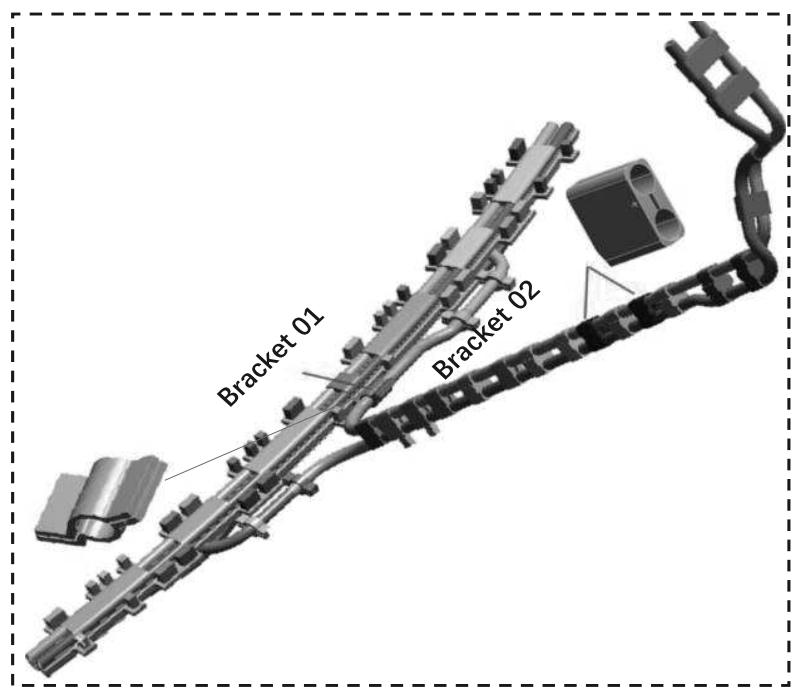

Fig. 14 The optimized VS coil

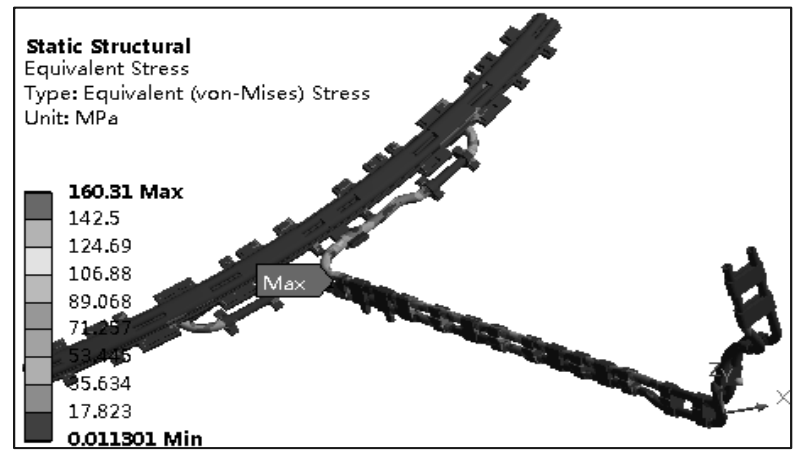

Fig. 15 The stress on the optimized VS coil

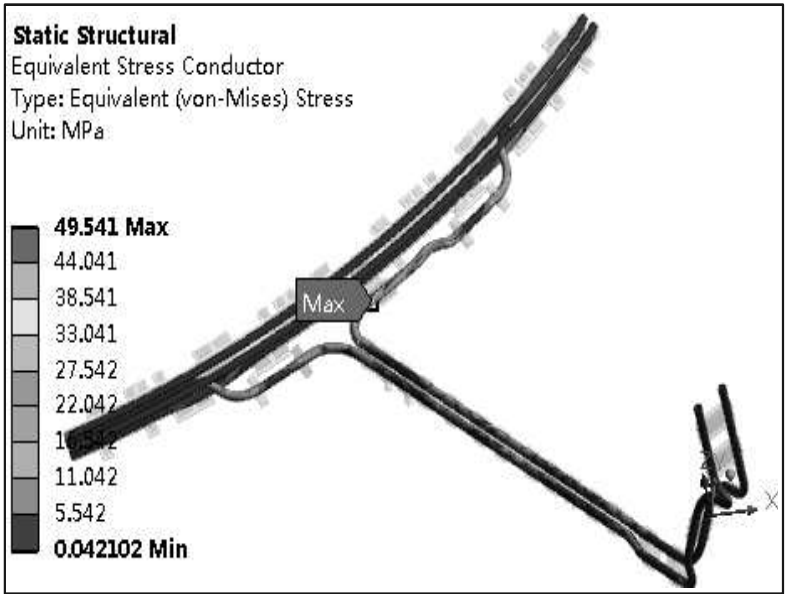

Fig. 16 The stress on the optimized copper conductor

\subsection{The stress evaluation based on analytical design}

Different methods can be used to carry out the stress evaluation [12]. Where the stress evaluation is based on ASME VIII-2 Part 5 analytical design. The stress is first classified into different types based on the external loads and then evaluated according to specific threshold. For the electromagnetic load, it mainly generates the primary general membrane stress, the local membrane stress and the bending stress. The general membrane stress is not allowed to beyond the design stress intensity. The design stress intensity for the copper conductor and stainless steel are $30 \mathrm{MPa}$ and $114 \mathrm{MPa}$ respectively. The local membrane stress and bending stress are not permitted to larger than 1.5 times of the design stress intensity. Before launching stress evaluation, stress linearization is done to extract the different kinds of stress on the VS coil components. The detail stress is listed in Table 2. It seems that most of the components satisfy the stress evaluation requirements. Except the total stress on the copper conductor is slightly larger than the threshold. During the engineering fabrication the fillets will be added on the bracket, which is helpful to decrease the total stress on the copper conductor.

Tab. 2 Stress evaluation of VS coil components

\begin{tabular}{cccccccc}
\hline Stress type (MPa) & $\begin{array}{c}\text { Copper } \\
\text { tube }\end{array}$ & Threshold & Jacket & Sleeve & Spine & Rail & Threshold \\
\hline General membrane & 28 & 30 & 90 & 98 & 16 & 45 & 114 \\
Membrane plus & 34 & 45 & 99 & 143 & 29 & 66 & 171 \\
bend & 50 & 45 & 148 & 160 & 35 & 67 & 171 \\
Total stress & & & & &
\end{tabular}

\section{Conclusion}

The magnetic field calculation results indicate the coil-body has higher average magnetic field than the feeder. But not all the magnetic field results in electromagnetic force, which is demonstrated by the fact that the electromagnetic force on the feeder is higher than that on the coil-body. It also indicates the toroidal field coil has more contribution on the electromagnetic force because it is nearer to the VS coil feeder. And due to the existence of $\mathrm{S}$ bending and $\mathrm{W}$ bending arcs, which lead to the electromagnetic force on the coilbody is not constant along the circumferential di- 
rection. According to the distribution of electromagnetic force on the feeder, it can be seen the parallel bonded design of feeder is a good way to counteract the opposite electromagnetic force. While for the break-out feeder, it is vulnerable to be damaged owing to the single feeder structure. The structural analysis indicates the break-out region should be reinforced. And by adding extra supporting bracket or increasing the size of bracket can effectively decrease the equivalent stress. However, based on the analytical design, the stress on the copper conductor is still a little higher than the allowable threshold. On the condition that the size of SSMIC is not allowed to be changed, the supporting components need to be further optimized to decrease the stress on the copper conductor. In fact, during the fabrication process some fillets will be machined on the supporting components, which is helpful to mitigate the local stress concentration.

\section{Acknowledgment}

This project has been financially supported by the Natural Science Foundation of China (Grant No. 51805230), the Applied Basic Research Program of Changzhou (Grant No. J20200011), the Natural Science Foundation of the Jiangsu Higher Education Institutions of China (Grant No. 19KJB470011).

\section{References}

[1] C. NEUMEYER, A. BROOKS, L.BRYANT, et al. (2011). Design of the ITER in-vessel coil. In: Fusion science and technology, Vol. 60, No. 1, pp. 95-99. AMER. USA.

[2] M. KALISH, P. HEITZENROEDER, A. BROOKS, et al. (2011). ITER in-vessel coil design and R\&D. 24th IEEE/NPSS Symposium on Fusion Engineering (SOFE), 2011 June 26-30, Chicago, USA.

[3] XIANEWEI WANG, ZHAOLIANG WANG, FEI XIE, et al. (2019). Electromagnetic Analysis of the Updated Fast Control Coil for EAST. In: Manufacturing Technology, Vol. 19, No. 1, pp. 172-176. Engineering Village. USA.

[4] H. JIN, Y. WU, F. LONG, et al. (2013). Investigation and analysis on ITER In-Vessel coils' raw-materials. In: Fusion Engineering and Design, Vol. 88, No. 11, pp. 3028-3032. Elsevier. Netherlands.

[5] LONG FENG, WU YU, DU SHIJUN et al. (2013). Manufacture of EAST VS In-Vessel Coil. In: Fusion engineering and design, Vol. 88, No. 12, pp. 3194-3198. ELSEVIER. Netherlands.

[6] E. F. DALY, K. IOKI, A. LOARTE et al. (2013). Update on Design of the ITER In-Vessel Coils. In: Fusion Science and Technology, Vol. 64, No. 2, pp. 167-175. Taylor \& Francis. USA

[7] R. T. HONJO, R. M. DEL VECCHIO. (1990). A program to computer magnetic fields, force, and inductances due to solid rectangular conductors arbitrarily positioned in spaces. In: IEEE Transactions on Magnetics, Vol. 22, No. 6, pp. 1532-1535. IEEE. USA

[8] LAXMIKANTK, URANKAR. (1982). Vector Potential and Magnetic Field of Current-Carrying Finite Arc Segment in Analytical Form. 3. Exact Computation for Rectangular Crosssection. In: IEEE Transactions on Magnetics, Vol. 18, No. 6, pp. 1860-1867. IEEE. USA.

[9] YUESEN CHU. (1999). Numerical Calculation for the Magnetic Field in Current-Carrying Circular Arc Filament. In: IEEE transactions on magnetics, Vol. 27, No. 6, pp. 1588-1595. IEEE. USA.

[10] ZHANG SW, SONG YT, WANG ZW, et al. (2014). Mechanical Analysis and Optimization of ITER Upper ELM Coil \& Feeder. In: Plasma Science \& Technology. Vol. 16, No. 8, pp. 794-799. IOP. England.

[11] SONTAMINO, ARKARAPON, PHANITWONG, WIRIYAKORN. (2017). Finite element analysis of counterbore-shaped parts by using sheet-bulk metal forming process. In: Manufacturing Technology, Vol. 17, No. 4, pp. 597-602. Engineering Village. USA.

[12] SESHADRI, R. (1996). Robust Stress-classification of pressure components using the GLOSS and GLOSS R-Node methods. In: Journal of Pressure Vessel Technology, Vol. 118, No. 2, pp. 208-215. ASME. USA 